\title{
Basis Functions for Estimating Intra-voxel Structure in DW-MRI
}

\author{
Alonso Ramírez-Manzanares, Mariano Rivera, Baba C. Vemuri and Thomas Mareci
}

\begin{abstract}
We present a new method for estimating and recovering the intra-voxel fiber paths, using Diffusion Weighted Magnetic Resonance Images (DW-MRI). The method recovers the intra-voxel information at voxels that contain fiber crossings or bifurcations by means of a combination of a known tensor basis functions (a "multi-tensor" field). In contrast with the stateof-the art methods, our formulation requires a small number of DWMR images and the solution schema is simple. Another advantage is that the solution to our formulation is numerically stable when more than two fiber orientations are present within a voxel. Additionally, we apply a spatial regularization to the multi-tensor field being estimated in order to denoise the data. The regularization uses a generic piece-wise smooth prior on the fiber orientation. Several examples are presented to demonstrate the performance of the proposed algorithm on synthetic and real DW-MRI data.
\end{abstract}

Index Terms-Multi-tensor MRI, Brain fiber tractography, Intra-voxel structure, DW-MRI, HARD DWI, DT-MRI.

\section{INTRODUCTION}

A MONGST the most challenging goals in neuroimaging is the estimation of connectivity patterns in the brain in vivo. For this purpose, a special magnetic resonance imaging (MRI) technique named Diffusion Weighted Magnetic Resonance Imaging (DW-MRI) is used. In this technique, it is possible to obtain an estimation of the orientation of water diffusion in a tissue specimen. Specifically in the brain, such diffusion is constrained by the direction of nerve bundles. This information is very useful in neuroscience research, due to the relationship of brain connectivity with several diseases and, in general, with brain development [1], [2]. In the recent past, there has been a flurry of activity on denoising the diffusion weighted MRI, in its tensor representation form [3]-[8]. Other notable works, involving diffusion tensor field segmentation can be found in [9]-[12] and involving tensor field registration in [13], [14].

Manuscript received May 19, 2004; revised June 18, 2004.

A. Ramirez is with the Computer Science Department, Centro de Investigacion en Matematicas A.C., Apdo. Postal 402, Guanajuato, Gto., 36000, Mexico. (telephone: (52) 47373271 55, alram@cimat.mx).

M. Rivera is with the Computer Science Department, Centro de Investigacion en Matematicas A.C., Apdo. Postal 402, Guanajuato, Gto., 36000, Mexico. (telephone: (52) 47373271 55, mrivera@ cimat.mx).

B.C. Vemuri is with Department of Computer Information Science and Engineering, University of Florida, Gainesville, Fl. 32611, USA. (vemuri@cise.ufl.edu),

T. Mareci is with Department of Biochemistry \& Molecular Biology, University of Florida, Gainesville, Fl. 32611, USA. (thmareci@ufl.edu).

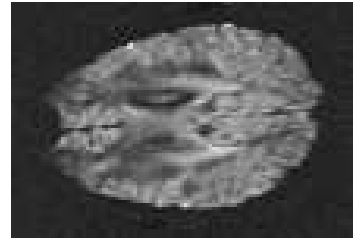

Fig. 1. An axial slice from a DW-MR brain scan for a single 3D orientation $\mathrm{g}_{i}$. The dark zones correspond to sites where the water diffusion is more significant, see text.

\section{A. Diffusion Tensor MRI}

The angular variation in the water diffusion has been summarized by the Diffusion Tensor Magnetic Resonance Imaging DT-MRI [15], [16]. The physical relationship of the water diffusion process for each image position $r$, in a given orientation, was established by the Stejskal-Tanner [17]:

$$
S_{i r}=S_{0 r} \exp \left(-b \mathbf{g}_{i}^{T} \mathbf{D}_{\mathbf{r}} \mathbf{g}_{i}\right)+\eta_{i r}
$$

where $S_{0 r}$ is the measured signal magnitude without diffusion gradient, $S$ is the attenuated signal in the tissue and $b$ is a constant directly proportional to the applied time and magnitude of the directional gradients. The unitary vector $\mathbf{g}_{i}=\left[g_{x}, g_{y}, g_{z}\right]^{T}$ indicates the $i-t h$ direction in which a directional independent magnetic gradient is applied, $\eta_{i r}$ represents a residual (generally produced by noise) and the diffusion coefficients in all directions are summarized by the positive definite symmetric $3 \times 3$ tensor $\mathbf{D}_{r}$. A standard acquisition protocol for a single orientation $\mathbf{g}_{i}$, gives a 3D image, where in each voxel $r$, the intensity indicates the grade of attenuation in the signal, lower gray value indicates larger attenuation ( $S$ is smaller) and this indicates significant water diffusion in the configured orientation, see figure 1.

The standard way to estimate $\mathbf{D}_{r}$ is by a Least Squares (LS) approach, i.e. given $S_{0 r}$ (a standard $T 2$ image [1]) and at least six measures $S_{i r}, i=1, \ldots, 6$ taken in different orientations, then the linear system (for the six unknown tensor components) generated by the $\log$ of (1) is solved. Note that this solver constrains that the tensor solution to be symmetric, but it does not constraint $\mathbf{D}$ to be positive definite. The diffusion tensor can be visualized as a 3D ellipsoid, with the principal axis aligned along the eigenvectors, $\left[\hat{e}_{1}, \hat{e}_{2}, \hat{e}_{3}\right]$, and scaled by the eigenvalues, $\lambda_{1} \geq \lambda_{2} \geq \lambda_{3}$. This tensor defines the diffusion magnitude along each axis. Thus, $\hat{e}_{1}$ is named the principal diffusion direction (PDD). A study of the behavior of the eigenvalues gives more 


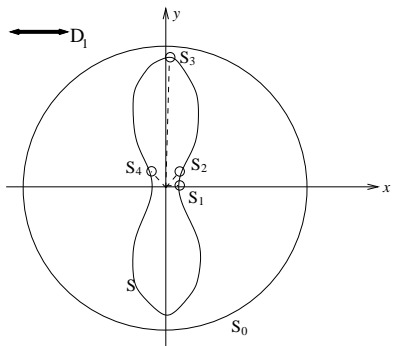

a)

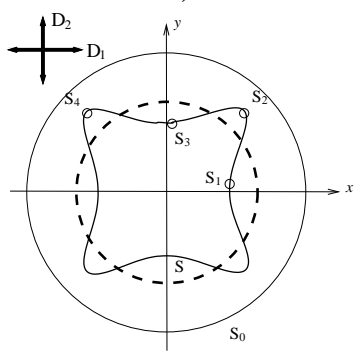

c)

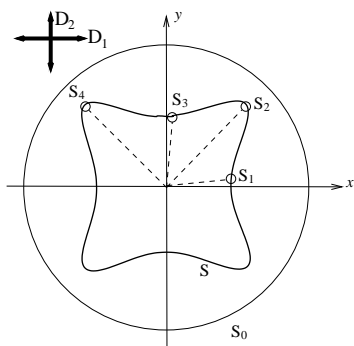

b)

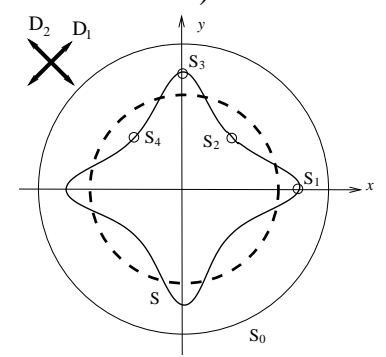

d)
Fig. 2. Schema of 3 different cases in DT-MRI. a) The signal $S$ (continuous ellipsoid) when there is only one water diffusion orientation $D_{1}$ in a voxel. b) The shape of $S$ (continuous line) when there are 2 almost orthogonal diffusion directions $D_{1}$ and $D_{2} . c$ ) and d) The shapes of the signal generated with the fitted tensor (dashed line) is the same in 2 different cases, see text.

insight on the diffusion angular variation into the voxels. In the $3 \mathrm{D}$ case, the measure of anisotropy most commonly used is the fractional anisotropy (FA) [16], [18], $F A(\mathbf{D})=$ $\sqrt{\left(\lambda_{1}-\lambda_{2}\right)^{2}+\left(\lambda_{1}-\lambda_{3}\right)^{2}+\left(\lambda_{2}-\lambda_{3}\right)^{2} / 2\left(\lambda_{1}^{2}+\lambda_{2}^{2}+\lambda_{3}^{2}\right)}$, where $\lambda_{k}$ is the $k^{\text {th }}$ eigenvector of $\mathbf{D}$. Note that for highly a anisotropic tensor, FA is close to one, while FA is close to zero for a low anisotropic tensor (the spherical case). Like in any other imaging technique, DT-MR images are corrupted by noise, and therefore, a denoising process is required.

\section{B. The partial volume problem}

Noise is not the only problem in DT-MR images; partial volume voxels have a more pronounced effect than in standard MR images. For instance, the observed diffusion tensor at voxels where two or more fibers cross, split, or merge, is the average of the diffusion in the constituent fiber directions. In most cases, the fitted single tensor model inadequately represents this information, because it is incapable of representing more than one diffusion direction. In order to understand this, we refer to the example in a plane illustrated in figure 2 . We illustrate 3 cases, where the continuous signal $S$ is drawn as a continuous line, the set of measurements $S_{i}, i=1, \ldots, 4$ are marked with the small circles, and the signal $S_{0}$ is represented with the big circle (since it is constant for every angle). In panel $2 \mathrm{a}$ we can observe a schema of the measured signal $S$ when there is only one diffusion direction, $D_{1}$, aligned with the $x$ axis. As we can see, the measured signal $S_{1}$ is the smallest one because the attenuation is biggest along that orientation. In this case, the fitted tensor explains the phenomena quite well. Panel $2 \mathrm{~b}$ illustrates the case when there are 2 diffusion orientations, almost orthogonal in a single voxel; the diffusion orientations $D_{1}$ and $D_{2}$ are aligned with the $x$ and $y$ axis, respectively. In this case, the maximal attenuations are in the $x$ and $y$ orientations. The LS method, would find the best tensor that explains the 4 measures. The resultant is a isotropic tensor that reproduces the dashed line shown in panel 2c. On the other hand, panel $2 \mathrm{~d}$ shows the case with two different orthogonal diffusion orientations, such that the fitted tensor is the same. Then, from the fitted tensor, we can not distinguish which of the previous cases have generated it.

So, the presence of two almost orthogonal tensors with highanisotropy, results in a fitted tensor with low-anisotropy. This fact increases the uncertainty of the tissue orientation (i.e., the inverse problem is not well-defined) [17], [19]. Moreover, the partial volume effect produced by fiber crossing can not be diminished by increasing the spatial resolution. The loss of directional information in fiber crossings and splits affects the estimation of fiber pathways, since, all the methods in literature that use DTI, follow the tensor field PDD in order to infer the fiber pathways [3][18][20]-[23]. Therefore, in order to compute a good estimation of the underlying fiber pathways, it is necessary to develop a process that recovers the lost intravoxel information, i.e., to develop a method that solves the limitations of the single tensor model.

\section{The intra-voxel information}

Although DT-MRI has seen a flurry of research activity in the recent past, the partial voluming affect has motivated the development of new ways of acquiring the data that can solve such problems. As a solution, Tuch et al. [24]-[26] proposed a high angular resolution diffusion imaging (HARDI) method, based on an observation model built from a finite mixture of Gaussians:

$$
S_{i}=S_{0} \sum_{j=1}^{M} \beta_{j} \exp \left(-b g_{i}^{T} R_{j}^{T} \Lambda R_{j} g_{i}\right)
$$

where $\Lambda$ is a constant diagonal matrix (i.e. the eigen-values were fixed). The unknowns, in (2), are: the $\beta$ coefficients and the rotation angles in $R$. These unknowns must be computed, independently for each voxel from a large set of acquired images $\{S\}$. This Diffusion Multi-Tensor Magnetic Resonance Imaging (DMT-MRI) technique allows one to recover the intravoxel information that is not observed in the standard DT-MRI. This imaging method is currently considered the state-of-theart in diffusion imaging. The drawbacks of the method are: the large number of additional diffusion images $\{S\}$ required (for instance, 126 diffusion 3D-images are used in [26], 54 in [27]), leading to large acquisition time, and the algorithmic problems related to Equation (2); which is highly nonlinear. So, multiple restarts of the optimization method are required to prevent the algorithm from settling in a local minima. Furthermore, no stable solution has been reported for more than 2 fiber bundles, i.e. for $j>2$ (see discussion on Ref. [26], Chap. 7).

Recently, a regularization method was proposed for solving the inverse problem in DT-MRI [28] i.e., a method for recovering the underlying structure/geometry within a voxel. 
This method assumed that the observed tensors are a linear combination of a given tensor basis. Therefore, the aim of their approach was to compute the unknown coefficients of this linear combination. Regularization of the problem requires prior assumption on the piecewise smoothness of nerve bundle orientations. Their reported results were quite good, however their multi-tensor model was applied to diffusivity computed from signal measurements using a uni-tensor model. In this paper we propose to directly use the raw measures $S_{i}$ (instead of the DTs) as input data. The aim is to avoid the information lost in using the uni-tensor model (given by the classical Stejskal-Tanner equation) to estimate the diffusivity.

The method we present here for reconstructing the intravoxel information uses a few diffusion weighted images, i.e., the intra-voxel information can be inferred at a fraction of the acquisition time required when using the HARDI data. Additionally, the proposed method is based on the minimization of a quadratic cost function (with non-negativity constraints) that can be efficiently minimized with standard deterministic algorithms, in particular we used an iterated Gauss-Seidel scheme.

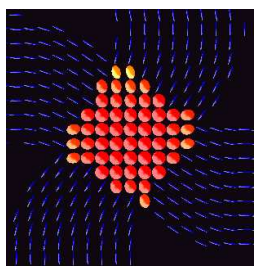

a)

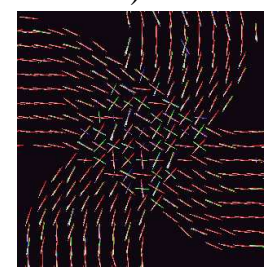

d)

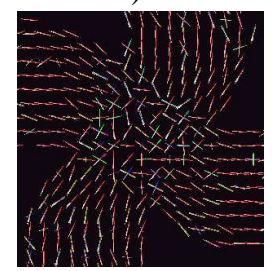

g)

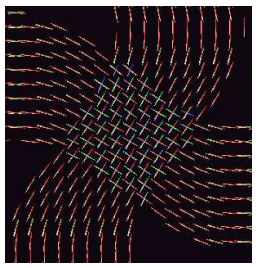

b)

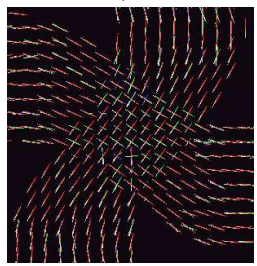

e)

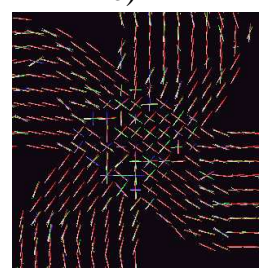

h)

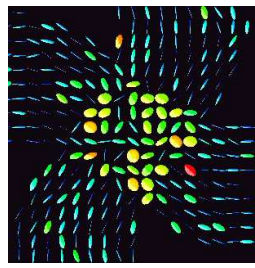

c)

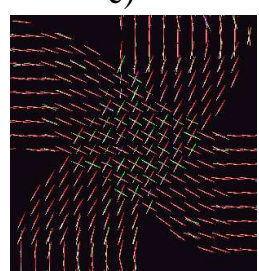

f)

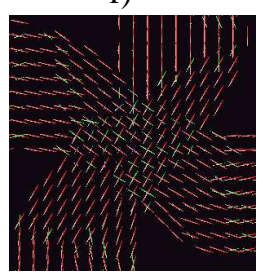

i)
Fig. 3. Synthetic results, see text and Table I.

\section{RECOVERING INTRA-VOXEL STRUCTURE USING A TENSOR BASIS}

In order to simplify the solution of (2) we propose to use a Base of Diffusion Functions (DFB) $\Phi$, which is generated from the same tensorial basis used in [28]. This tensor basis, that generates the DFB, is defined as a fixed set of tensors $\overline{\mathbf{T}}$ with cardinality equal to $N$. The individual base tensors $\overline{\mathbf{T}}_{j}$ are chosen, such that, they are uniformly distributed on the 3D space of orientations, and its $\operatorname{FA}\left(\overline{\mathbf{T}}_{j}\right) \approx 1$ is chosen according the prior information that the diffusion along the fiber is five times that of in the transversal orientations $\left[\lambda_{1}, \lambda_{2}, \lambda_{3}\right]=$ $[1 E-3,2 E-4,2 E-4]$. Using the DFB, the model that we propose for each $r$ position, given $S_{0}$, is:

$$
S_{i r}=S_{0 r} \sum_{j=1}^{N} \alpha_{j r} \Phi_{i j}+\eta_{i r}
$$

with $\alpha_{j r} \geq 0$; where we define the diffusion basis function by:

$$
\Phi_{i j}=\exp \left(-b g_{i}^{T} \overline{\mathbf{T}}_{j} g_{i}\right)
$$

where each $\Phi_{i j}$ should be understood as the decay factor due to the base tensor $\overline{\mathbf{T}}_{j}$ in the $i$-th direction $g_{i}$. The $\alpha=$ $\left[\alpha_{1 r}, \alpha_{2 r}, \ldots, \alpha_{j r}, \ldots, \alpha_{N r}\right]^{T}$ in 3 is a vector field, such that the scalar $\alpha_{j r}$ denotes the contribution of the individual basis function $\Phi_{i j}$ to all the $i$ gradient directions, i.e. the contribution to the Gaussian mixture at voxel $r$. Note that the DFB is, in general, not complete, due to the fact that the available orientations are a discretization of the $3 \mathrm{D}$ space, leading to a remanent scalar (generated by the model in (3)) for each measurement denoted by $\eta_{i r}$. The chosen basis cardinality can diminish this remainder, until it becomes insignificant and is mainly attributed to the noise in the signal.

The significant advantage of our proposed model is that the $\Phi_{i j}$ basis function in (4) can be precomputed, because the gradients, the basis tensors and the $b$ value are known a priori, and therefore, the task consists only in finding the $\alpha_{r}$ vector in each position. In fact, we need to find the best combination of basis tensors that reproduce the set of measured $S_{i}$ 's. Figure 2 illustrates this: we need to find the $\alpha$ values that, given all the $\Phi_{i j}$ functions reproduces the $S_{i}, i=1, \ldots, 4$ measurements as best as possible. In order to find the $\alpha_{r}$ vector, we propose to minimize the following cost function:

$$
U(\alpha, r)=\sum_{i=1}^{M}\left(S_{i r}-S_{0} \sum_{j=1}^{N} \alpha_{j r} \Phi_{i j}\right)^{2},
$$

subject to $\alpha_{j r} \geq 0$; where $M$ is the number of measures, i.e. the number of different magnetic gradients. Equation (5) takes in to account the differences between the $S_{i}$ measured signals and the proposed model in (3). The partial differentiation of (5) with respect a each $\alpha_{j r}$ give us a constrained linear system, that can be solved easily by a Gauss-Saidel (GS) scheme, that is memory efficient. The positivity restriction over the $\alpha$ coefficients establishes $N$ constraints in the minimization problem. This GS is a particular case of the well known Gradient Projection: the $\alpha_{j r}$ values are projected to zero in each iteration.

Regularizing the solution The adverse effect of noise could possibly preclude the original diffusion directions. In our model, the noise could lead to estimated $\alpha$ coefficients that do not correspond to the correct diffusion orientations or they may not indicate the right number of diffusion orientations in each voxel. In order to diminish the noise effect, we propose to filter the $\alpha$ coefficients. For this task, we use prior information about the piecewise smoothness of nerve bundles orientation. Since each $\alpha_{j r}$ coefficient is implicity associated to a diffusion 
TABLE I

MEAN ANGULAR ERROR $\bar{\epsilon}$ FOR THE EXPERIMENTS IN FIG. 3.

\begin{tabular}{llllll}
\hline Panel & Method & Noise $\sigma$ & $\lambda_{s}$ & $\lambda_{c}$ & $\bar{\epsilon}$ \\
\hline a) & LS & 0 & - & - & - \\
b) & our & 0 & 0.0 & 0.0 & 1.48 \\
c) & LS & 0.2 & - & - & - \\
d) & our & 0.1 & 0.0 & 0.0 & 4.49 \\
e) & our & 0.1 & 0.028 & 0.002 & 2.29 \\
f) & our & 0.1 & 0.05 & 0.009 & 6.42 \\
g) & our & 0.2 & 0.0 & 0.0 & 6.69 \\
h) & our & 0.2 & 0.028 & 0.002 & 4.68 \\
i) & our & 0.2 & 0.12 & 0.009 & 6.85 \\
\hline
\end{tabular}

orientation, the spatial smoothness of each $\alpha_{j}$ is mutually related with a spatial smoothness of the $j-t$ th diffusion direction. We use the same filtering schema that is used in work [28], where the spatial smoothness is achieved by the regularization term: $U_{s s}(\alpha, r)=\sum_{s: s \in \mathcal{N}_{r}} \sum_{j} w_{j r s}\left(\alpha_{j r}-\alpha_{j s}\right)^{2}$, which promotes a small difference between the $\alpha$ vector in the $r$ position and the vectors $\alpha_{s}$ that are in $r$ 's neighborhood. The spatial smoothness for each $\alpha_{j}$ layer is constraint along the PDD of the $\overline{\mathbf{T}}_{j}$ base tensor, this is achieved by the use of the factor $w_{j r s}=(s-r)^{T} \overline{\mathbf{T}}_{j}(s-r) /\|s-r\|^{4}$, where $w_{j r s}$ is the weight associated with the potential $\left(\alpha_{j r}-\alpha_{j s}\right)^{2}$, see [28].

Finally we use a filtering term that promotes large contrast in the $\alpha_{j r}$ coefficients. We want a large contrast in the coefficients in order to clearly discern which of the coefficients (orientations) are representative and which of them has a value different from zero because of the noise or the discrete nature of the basis tensor. This is achieved by forcing each $\alpha_{j r}$ coefficient to be different from the arithmetic mean: $\bar{\alpha}_{r}=\sum_{j} \alpha_{j r} / N$, see [28].

\section{MinimizATION}

The final cost function to be minimized is:

$$
\begin{array}{r}
U(\alpha, r)=\sum_{i=1}^{M}\left(S_{i r}-S_{0} \sum_{j=1}^{N} \alpha_{j r} \Phi_{i j}\right)^{2}+ \\
\lambda_{s} \sum_{s: s \in \mathcal{N}_{r}} \sum_{j} w_{j r s}\left(\alpha_{j r}-\alpha_{j s}\right)^{2}-\lambda_{c} \sum_{j}\left(\alpha_{j r}-\bar{\alpha}_{r}\right)^{2},
\end{array}
$$

subject to $\alpha_{j r} \geq 0$. As can be seen, because all the potentials in (6) are quadratic, the constrained minimization procedure is reduced again to the solution of a linear system in the unknowns $\alpha$ with the projection to zero of $\alpha_{j r}$ in each iteration.

\section{RESULTS}

In order to evaluate the proposed algorithm, we develop a synthetic validation procedure. First, we simulated two wavy fiber crossings, and for each voxel, we established the diffusion coefficients $D(1$ or 2$)$ and the diffusion orientation for each fiber. Then, we computed the $S_{i},(i=1, \ldots, 4)$ coefficients according to the expression in [1]. The expressions for the case of one and two directions of diffusion are respectively given by: $S_{i}=S_{0} e^{-b\left(D_{1} \cos ^{2} \theta_{i}+D_{2} \sin ^{2} \theta_{i}\right)}$ and $S_{i}=S_{0} e^{-b\left(D_{1} \cos ^{2} \theta_{i}+D_{2} \sin ^{2} \theta_{i}+D_{3} \cos ^{2} \phi_{i}+D_{4} \sin ^{2} \phi_{i}\right)}$. Where $D_{1}, D_{2}$ are the principal and secondary diffusion coefficients

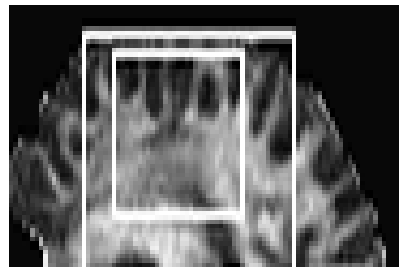

a)

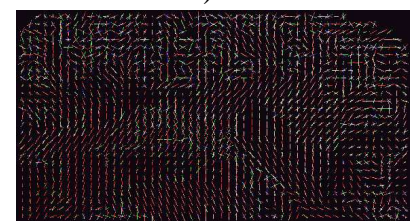

c)

e)

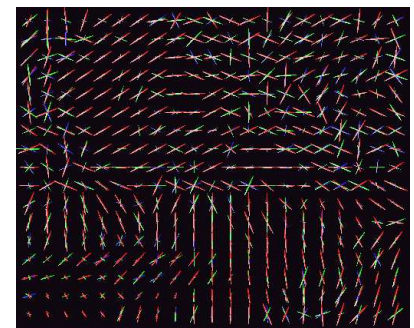

g)

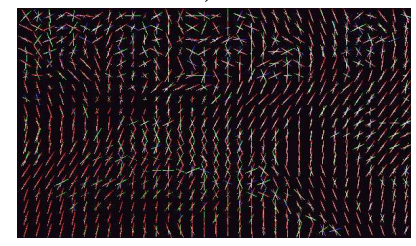

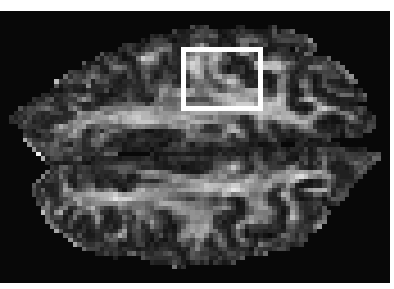

b)

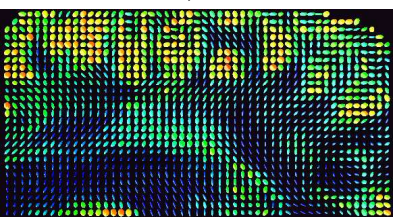

d)

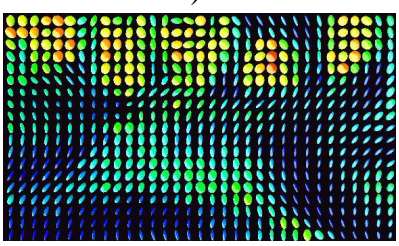

f)

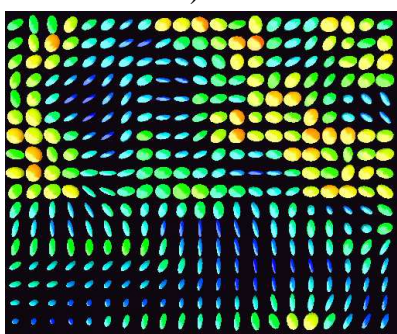

h)
Fig. 4. Results of experiments with brain DWI, see text.

respectively for the first fiber and $D_{3}, D_{4}$ are the principal and secondary diffusion coefficients respectively for the second fiber $\left(\frac{D_{1}}{10} \approx \frac{D_{3}}{10} \approx D_{2} \approx D_{4}\right)$. $\theta_{i}$ is the angle between the orientation of the first fiber and the $i$-th acquisition axis, $\phi_{i}$ is the same angle but for the second fiber, see [1]. The $b$ coefficient was set to $1000 \mathrm{~s} / \mathrm{mm}^{2}$ according to the standard DTMRI protocol, and $D_{1}=1^{-3} \mathrm{~mm}^{2} / \mathrm{s}$ (a typical number for the brain). Next we fitted a tensor in each voxel by a Least-Square method [29]. We obtained the multi-tensor field according to the method described in section II with the basis cardinality $N=30$, and finally we measured the angle difference, $\epsilon$, between the original diffusion orientations and the recovered multi-tensors PDD's. The mean angular error and the references to the figures are in table I. We can note that the regularization process eliminates the noise effect, but the smoothness in the trajectories is affected. However, this effect is not too pernicious when we are inferring fiber pathways (about 6 degrees in the worst case).

The results obtained with brain DW-MRI are shown in figure 4. In this case, we used the DW images that are included in the BioTensor software, provided by the web site of the Scientific Computing and Imaging Institute of the 
University of Utah (http: / / software.sci.utah.edu/ archive/archive_main.html). Which are constructed to $2.0 \times 2.0 \times 2.0 \mathrm{~mm}^{3}$, and have volume dimensions of $75 \times 109 \times 29$. The DW data have 12 measurements per voxel in independent directions $g_{i}, b=1000$. In this experiment the regularization parameters $\lambda_{s}$ and $\lambda_{c}$ were not used, because the low noise level in the data. The tensor basis was composed by 33 equidistant tensors. Panel $4 \mathrm{a}$ and $4 \mathrm{~b}$ depicts the FA map with the interest regions marked by white squares. The big square in panel 4a (a sagital slice) corresponds to the panels $4 \mathrm{c}$ and $4 \mathrm{~d}$ respectively. The small square in panel $4 \mathrm{a}$ (an axial slice) corresponds with the panels $4 \mathrm{e}$ and $4 \mathrm{f}$ respectively. Finally the square in panel $4 \mathrm{~b}$ corresponds with the panels $4 \mathrm{~g}$ and $4 \mathrm{~h}$ respectively. We show for each square the computed multi-tensor field and the recovered tensor field using $\mathbf{D}_{r}^{f}=$ $\sum_{j} \alpha_{j} \overline{\mathbf{T}}_{j}$, i.e., by reconstructing a tensor by the sum over the contributions of each base tensor. We note that the results, in panels $4 \mathrm{e}$ and $4 \mathrm{~g}$, are congruent with the a priori anatomical knowledge for those regions, in particular, panel 4 a corresponds to the corona radiata.

\section{CONCLUSIONS}

In this paper, we presented a new multi-tensor representation that was used to directly obtain the local nerve bundle geometry from diffusion weighted MR measurements. The key contribution is that our formulation for inferring the local geometry of the nerve fibers, allows us to infer complicated local fiber geometry with DWIs collected along very few gradient directions as opposed to techniques using HARDI data sets. Also, another key advantage is that our multi-tensor model leads to a constrained quadratic optimization problem which is minimized, very efficiently, by solving a bounded linear system. Finally, the proposed method is very efficient as the tensor basis used in the multi-tensor model can be pre-computed. We presented examples of performance of our algorithm on synthetic and real data sets and in the former case, the results were validated.

\section{ACKNOWLEDGEMENT}

The authors were supported in part by CONACYT, Grant P40722Y1 (Ramirez-Manzanares and Rivera), and by NIH, Grant RO1 NS42075 (Vemuri and Mareci).

\section{REFERENCES}

[1] Buxton Richard. Introduction to Functional Magnetic Resonance Imaging Principles and Techniques. Cambridge: Cambridge University Press; 2002.

[2] Poldrack, R. A. (2001). A structural basis for developmental dyslexia: Evidence from diffusion tensor imaging, In M. Wolf (Ed.), Dyslexia, Fluency, and the Brain, 213-233.

[3] B. C. Vemuri, Y. Chen, M.Rao, T.McGraw, Z. Wang and T.Mareci, Fiber Tract Mapping from Diffusion Tensor MRI, IEEE Workshop on VLSM'01, $81,2001$.

[4] T. McGraw and B. C. Vemuri and Y. Chen and M. Rao and T. H. Mareci, DT-MRI denoising and neuronal fiber tracking Med. Image Anal. 8(2):95111,2004

[5] Z. Wang and B. C. Vemuri and Y. Chen and T. H. Mareci, A Constrained Variational Principle for Direct Estimation and Smoothing of the Diffusion Tensor Field from complex DWI, IEEE Trans, Med. Imag.,In press., 2004.
[6] D. Tschumperlé and R. Deriche, Variational frameworks for DT-MRI Estimation, regularization and visualization, Proc. 9th IEEE-ICCV, (2):116121, 2003.

[7] C. Chefd'hotel and O. Faugeras and D. Tschumperlé and R. Deriche, Constrained flows of matrix-valued functions: application to diffusion tensor regularization, Proc. 7th ECCV '02, Springer LNCS, (2351):251$265,2002$.

[8] J. Weickert, Diffusion and regularization methods for tensor-valued images, First SIAM-EMS Conf. Appl. Math. in Our Changing World, 2001.

[9] Z. Wang and B. C. Vemuri, Tensor Field Segmentation Using Region Based Active Contour Model, Proc. of the 8th ECCV'04 (4), Springer LNCS, (3024):304-315, 2004.

[10] Z. Wang and B. C. Vemuri, An affine invariant tensor dissimilarity measure and its applications to tensor-valued image segmentation, IEEE CVPR'04,In press., 2004.

[11] C. Lenglet and M. Rousson and R. Deriche, Toward Segmentation of $3 D$ probability Density Fields by Surface Evolution: Application to Diffusion MRI, 7th MICCAI'04,In press., 2004.

[12] L. Zhukov, K. Museth, D. Breen, R. Whitaker and A. Barr, Level Set Modeling and Segmentation of DT-MRI Brain Data, J. Electronic Imaging, Vol. 12, No. 1, January 2003, pp. 125-133

[13] J. Ruiz-Alzola, C.-F. Westin, S. K. Warfield, A. Nabavi, R. Kikinis: Nonrigid Registration of 3D Scalar, Vector and Tensor Medical Data, MICCAI 2000: 541-550

[14] J. C. Gee, D. C. Alexander, M. Rivera, J. T. Duda Non-rigid registration of diffusion tensor MR images, , IEEE ISBI'02, Washington D.C., IEEE Press, 477-480, Jul 2002.

[15] P.J. Basser, J. Mattiello, and D. Le Bihan, MR diffusion tensor spectroscopy and imaging. Biophys. J. 66(1), 259-67 (1994). 4.

[16] P.J. Basser and S. Pajevic and C. Pierpaoli and J. Duda and A. Aldroubi, Microestructural and phisiological features of tissues elucidated by quantitative-diffusion-tensor MRI, J. Magn. Reson., 111, 209-219, 1996.

[17] C.F. Westin, S.E. Maier, H. Mamata, F.A. Jolesz, R. Kikinis, Processing and Visualization for Diffusion Tensor MRI, Medical Image Analysis, 6(2):93-108, 2002.

[18] P.J. Basser and S. Pajevic and C. Pierpaoli and J. Duda and A. Aldroubi, In Vivo Fiber Tractography Using DT-MRI Data, Magn. Reson. Med.. 44 (2000) 625-632.

[19] Mette R. Wiegell, MSc, Henrik B. W. Larsson, MD, PhD, Dr med and Van J. Wedeen, MD Fiber Crossing in Human Brain Depicted with Diffusion Tensor MR Imaging, Radiology. 2000;217:897-903

[20] Basser PJ, Pajevic S, Pierpaoli C and Aldroubi A, Fiber tract following in the human brain using DT-MRI data, IEICE Trans Inf Sys, E85D, 15-21, 2002.

[21] L. Zhukov and A.H. Barr, Oriented Tensor Reconstruction: Tracing Neural Pathways from Diffusion Tensor MRI, Proceedings of IEEE Visualization 2002, 387-394, Oct. 2002.

[22] M. Björnemo, B. Anders White Matter Fiber Tracking Using Diffusion Tensor MRI, Master's Thesis, Linköping University, Reg nr: LiU-IMT-EX321, 2002.

[23] Lazar M, Weinstein DM, Tsuruda JS, Hasan KM, Arfanakis K, Meyerand ME, Badie B, Rowley HA, Haughton V, Field A, Alexander AL. White matter tractography using diffusion tensor deflection, Hum Brain Mapp. 2003 Apr; 18(4):306-21.

[24] D.S. Tuch, R.M. Weisskoff, J.W. Belliveau, V.J. Wedeen, High angular resolution diffusion imaging of the human brain, Proc. 7th Annual Meeting of ISMRM, Philadelphia, 321, 1999.

[25] D.S. Tuch, T.G. Reese, M.R. Wiegell, N. Makris, J.W. Belliveau, Wedeen VJ. High angular resolution diffusion imaging reveals intravoxel white matter fiber heterogeneity, Magn. Reson. Med., 48(4), 577, 2002

[26] D.S. Tuch, Diffusion MRI of complex tissue structure, Doctoral Thesis, Harvard-MIT, Cambridge MA, 2002.

[27] G.J.M. Parker and D.C. Alexander. Probabilistic Monte Carlo Based Mapping of Cerebral Connections Utilising Whole-Brain Crossing Fibre Information, Proc. of IPMI'03, 684-695, 2003.

[28] A. Ramírez-Manzanares, M. Rivera Brain Nerve Bundles Estimation by Restoring and Filtering Intra-Voxel Information in Diffusion Tensor MRI, Procc. 2 IEEE Workshop on VLSM'03, 73-80, Oct 11-12, Nice, Fr. 2003

[29] C.F. Westin, S.E. Maier, B. Khidhir,P. Everett,F.A. Jolesz, R. Kikinis, Image Processing for Diffusion Tensor Magnetic Resonance Imaging, In: Taylor C, Colchester A, editors. MICCAI'99, Sep 9-22, Cambridge, England. Heidelberg, Germany: Springer Verlag, 441-452, 1999. 